Technical Report No. 6/08, December 2008 ACTUARIAL APPLICATIONS OF EPIDEMIOLOGICAL MODELS

Runhuan Feng and José Garrido 


\title{
Actuarial Applications of Epidemiological Models
}

\author{
Runhuan Feng ${ }^{1}$, José Garrido ${ }^{2}$ \\ ${ }^{1}$ Department of Mathematical Sciences \\ University of Wisconsin - Milwaukee, USA \\ ${ }^{2}$ Department of Mathematics and Statistics \\ Concordia University, Montreal, QC, Canada \\ fengr@uwm.edu, garrido@mathstat.concordia.ca
}

November 20, 2008 (revised February 17, 2010)

\begin{abstract}
The risk of a global avian flu or influenza A (H1N1) pandemic, and the emergence of the worldwide SARS epidemic in 2002-03 have led to a revived interest in the study of infectious diseases. Mathematical models have become important tools in analyzing the transmission dynamics and in measuring the effectiveness of controlling strategies. Research on infectious diseases in the actuarial literature only goes so far as to set up epidemiological models which better reflect the transmission dynamics. This paper attempts to build a bridge between epidemiological and actuarial modeling and set up an actuarial model which provides financial arrangements to cover the expenses resulting from the medical treatments of infectious diseases.

Based on classical epidemiological compartment models, the first part of this paper proposes insurance policies and models to quantify the risk of infection and formulates financial arrangements, between an insurer and insureds, using actuarial methodology. For practical purposes, the second part employs a variety of numerical methods to calculate premiums and reserves. The last part illustrates the methods by designing insurance products for two well known epidemics: the Great Plague in England and the SARS epidemic in Hong Kong.
\end{abstract}

Keywords: epidemiological compartment models; SIR model; actuarial mathematics; infectious diseases; health insurance; Runge-Kutta method; ratemaking; reserves.

JEL Classification: C02. 


\section{Introduction}

The Severe Acute Respiratory Syndrome (SARS) epidemic in 2002-03 drew tremendous attention to the treatment and prevention of infectious diseases and their impact on our society's welfare. The adverse economic impact caused by SARS in East Asia has often been compared with that of the 1998 financial market crisis in that area.

From a social point of view, an effective protection against diseases depends not only on the development of medical technology, to identify viruses and to treat infected patients, but also on a well-designed health-care system. The latter can reduce the financial impact of a sudden pandemic outbreak, such as surging costs of medications, hospital infrastructures and medical equipments, prevention measures like vaccination and quarantine. Broader insurance programs can even cover financial losses resulting from the interruption in regular business operations. As a profession with the reputation in applying mathematical techniques to model and quantify financial risk, actuaries are certainly well placed to expand their expertise and deal with epidemics within health-care systems.

Due to their front-line experience with SARS epidemic, many health insurers in Asia provided coverage to compensate for medical costs of SARS treatment, by listing the disease as an extended liability on regular health insurance policies. Still many problems arose. Traditional actuarial models for human mortality lack the flexibility required to model infectious diseases, which in many respects are significantly different from natural causes of death.

One of these remarkable differences is that in a population exposed to an epidemic outbreak there are several mutually dependent groups involved, with different levels of vulnerability to the disease. This contrasts with mortality rates that are often assumed to be constant among homogeneous age-specific groups.

How fast an infectious disease spreads within a population relies on the number of susceptible individuals, the number of infectious individuals and the social structure between these two groups. To be more specific, in the context of a health insurance for an initially complete susceptible group, the number of insureds bearing premiums would actually decrease in time, whereas the number of insureds claiming benefits due to infection increases as the epidemic breaks out. Applying traditional life table methods overlooks epidemiological dynamics and dependence between insurance payers and beneficiaries. It consequently violates the fair premium principle.

Other than the traditional life table methods, there have been developments in the recent actuarial literature of alternative multi-state Markov models. These partition a population according to the status the insured can be in; for instance alive, disabled, and dead. Multistate Markov models are well suited for traditional insurance products in which payments 
made differ according to the particular status an insured is in. For instance, death benefits are payable upon the death of an insured in life insurance, while annuities are payable as long as an insured stays alive. For detailed accounts of multi-state Markov models, readers can consult Waters (1984), Hoem (1988) and Jones (1994). While the multi-state model has its own merits in the mathematics of traditional insurance, we propose here actuarial calculations based on epidemiological models. We believe that these present some advantages in modelling insurance against infectious diseases, such as:

1. Compartment models in the epidemiology are constructed in accordance to the law of mass action widely used in many areas of biology, chemistry and physics. As alluded to earlier, the unique feature of the infectious disease, with its dynamics of transmission depending on the interactions of two subgroups of a population, can only be reflected in the utilization of the law of mass action.

2. There have been extensive studies and substantial empirical data analysis on the validity and parameter estimations of compartment models. Actuaries have also been involved and gained expertise in fitting of these models to data, as seen in Jia and Tsui (2005).

3. Epidemiological models can be used in sensitivity tests for prevention and intervention measures and hence can be used to analyze the impact on the financial obligations of insurance products for infectious diseases.

Therefore, we present in this paper a new approach that uses epidemiological models as building blocks, and develop a framework for actuarial calculations with applications to insurance coverages targeting infectious diseases.

To make the paper self-contained, Section 2 is devoted to a brief review of a simple model from the mathematics of epidemiology, the three-compartment model. An insurance model is developed to make arrangement between an insurer and insurance policy-holders in order to protect the insured from the potential financial burden resulting from infection by a disease.

To apply the ideas in an insurance context, Section 3 formulates the epidemiological model in standard actuarial notation and analyzes the quantitative relations among some insurance concepts, namely the actuarial present value of continuous payments for hospital and medical services, death benefits and premium income.

In Section 4, several ratemaking methods are presented for various infectious disease insurance policies. Thanks to an algorithm that calculates premiums under the fair premium principle, we then look at the solvency of these insurance plans. 
Since benefit reserves generally reflect a policy's cash value that is refundable to the policy-holders, it is expected for the benefit reserves to remain positive throughout the life of the policy. However, as one shall see, level net premiums lead to negative reserves, due to the distinct nature of infectious diseases. Therefore Section 5 analyzes the reasons behind the negative reserves and proposes a numerical method developed to determine adjusted premiums that keep benefit reserves from falling below a minimum tolerance level.

Based on models calibrated in the epidemiological literature, we analyze in Section 6 the dynamics of the Great Plague in Eyam, England and that of the SARS epidemic in Hong Kong. This leads to insurance policy designs to cover the resulting financial losses. The analytical procedures could easily be adapted to enable an analysis of a wide range of scenarios.

\section{Epidemiological compartment model}

Over the last century, many contributions to the mathematical modeling of infectious diseases have been made by a great number of public health physicians, epidemiological mathematicians and statisticians. Their brilliant work ranges from empirical data analysis to the theory of differential equations. Many have been applied successfully in clinical data analysis to make effective predictions. For a complete review of a variety of mathematical and statistical models, see Hethcote (2000) and Mollison et al. (1994). Building on the work of such pioneers, actuaries can add economical considerations to epidemiological models and design insurance policies which can provide financial means to protect the general public against the adverse economic impact of epidemics. For an account of existing co-operative opportunities for actuaries and epidemiologists, readers are referred to a report by Cornall et al. (2003). More recently epidemiological models have been used in applications to Entreprise Risk Management (ERM), see for instance Chen and Cox (2007), while pandemic risks are frequently discussed in actuarial circles, see Stracke and Heinen (2006) for influenza, Mäkinen (2009) for H1N1 and CIA (2009) for pandemic scenarios.

To illustrate the possible actuarial applications, we first look at a simple deterministic epidemiological model, which could lead to a straightforward actuarial analysis. Although most infectious diseases, like SARS, are far too complex to fit such a three-compartment model, the generalization to multi-dimensional models follows similar procedures. A detailed account of compartment models can be found in Anderson and May (1991), Brauer and Castillo-Chávez (2001) and Hethcote et al. (1981).

In epidemiological models, the whole population is usually separated into compartments for different individuals. They are often labeled by acronyms, such as $S, I$ and $R$, in 
different patterns according to the transmission dynamics of the studied disease. Generally speaking, class $S$ denotes the group of healthy individuals without immunity, or in other words, those who are susceptible to a certain disease or virus. In an environment exposed to the disease, some of these individuals come into contact with the virus. The individuals who are infected and able to transmit the disease are classified in class $I$. Through medical treatment, individuals, removed from the epidemic due to either death or recovery, are all counted in class $R$. The upper part of Figure 1 gives the transferring dynamics among the three compartments.

Another merit of this partition, from an actuarial perspective, is that the three compartments play significantly different roles in an insurance model. As shown in the lower part of Figure 1, susceptibles facing the risk of being infected in an epidemic form a market that could contribute premiums to an insurance fund, in return for the coverage for medical expenses incurred if infected. During the outbreak of an epidemic, the infected policy-holders would benefit from the claim payments provided by the insurance fund. Furthermore, following an insured individual's death, a death benefit for funeral and burial expenses may also be paid to the beneficiaries designated by the insured person. Once the insurance fund is set up, interest accrues at a certain rate on the unpaid reserves.

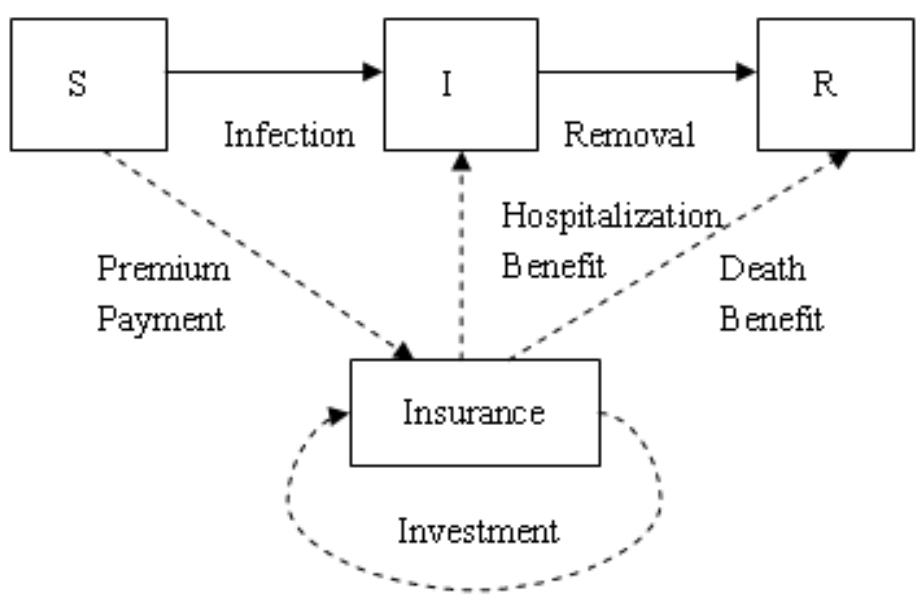

Figure 1: The transmission and insurance dynamics among compartments $S, I$ and $R$

We first look at a simple mathematical (SIR) model that characterizes the interaction among the three compartments. Let $S(t)$ denote the number of susceptible individuals at time $t$, whereas $I(t)$ is the number of infected and $R(t)$ the number of removed individuals from class $I$. According to the law of mass action which is commonly used in chemistry and biological studies, the rate of change of reactions is proportional to the concentration of participants. In the context of epidemiology, the rate of increase in the number of the 
infected is hence proportional to the number of susceptible individuals and the number of individuals previously infected. Translated into mathematical languages, the rate of changes in the size of compartments can be interpreted as respective derivatives. Therefore, the evolution of compartment sizes is driven by the following system of ordinary differential equations (ODE's) known as the SIR model:

$$
\begin{aligned}
S^{\prime}(t) & =-\beta S(t) I(t) / N, \quad t \geq 0, \\
I^{\prime}(t) & =\beta S(t) I(t) / N-\alpha I(t), \quad t \geq 0, \\
R^{\prime}(t) & =\alpha I(t), \quad t \geq 0,
\end{aligned}
$$

with given initial values $S(0)=S_{0}, I(0)=I_{0}, S_{0}+I_{0}=N$ and constant rates $\alpha \in[0,1]$, $\beta>0$. The model is based on the following assumptions:

1. The total number of individuals remains constant, $N=S(t)+I(t)+R(t)$, representing the total population size.

2. An average susceptible makes an average number $\beta$ of adequate contacts (i.e. contacts sufficient to transmit infection) with others per unit time.

3. At any time a fraction $\alpha$ of the infected leave class $I$ due to death and $\alpha$ is considered to be the fatality rate of the specific disease.

4. There is no entry into or departure from the population, except possibly through death from the disease. For our purpose of setting up an insurance model, the demographic factors like natural births and deaths are negligible, as the time scale of an epidemic is generally shorter than the demographic time scale.

Since the probability of a random contact by an infected person with a susceptible individual is $S / N$, then the instantaneous increase of new infected individuals is $\beta(S / N) I=\beta S I / N$. The third assumption implies that the instantaneous rate of death in the number of infected individuals is proportional to the current size of compartment $R$.

\section{Actuarial analysis}

The idea of insurance coverage against the financial impact due to infectious diseases is akin to that of coverage for other contingencies, like accidental death and destruction of properties. Yet it is distinctive in nature from property and casualty insurance, because the number of policy-holders bearing the premiums and the number of policy-holders eligible for compensations vary over time throughout the epidemic. 
Since mortality analysis is based on ratios instead of absolute counts, we now introduce the deterministic functions $s(t), i(t)$ and $r(t)$, interpreted respectively as the fractions of the population in each of class $S, I$ and $R$. Dividing equations (2.1)-(2.3) by the constant total population size $N$ yields

$$
\begin{aligned}
s^{\prime}(t) & =-\beta i(t) s(t), \quad t \geq 0, \\
i^{\prime}(t) & =\beta i(t) s(t)-\alpha i(t), \quad t \geq 0, \\
r(t) & =1-s(t)-i(t), \quad t \geq 0,
\end{aligned}
$$

where $s(0)=s_{0}$ and $i(0)=i_{0}$, given that $s_{0}+i_{0}=1$.

One can actually interpret the ratio functions $s(t), i(t)$ and $r(t)$ as the probability of an individual being susceptible, infected or removed from infected class respectively at the time $t$. However, it should be noted that due to the law of mass action, movements between the compartments depend on the relative sizes of one another. Thus these probabilities correspond to mutually dependent risks for the SIR model, as opposed to the usual independent hazards in multiple decrement life insurance models. With these probability density functions $s(t), i(t)$ and $r(t)$, we now incorporate actuarial methods to formulate the quantities of interest for an infectious disease insurance.

\subsection{Annuity premium and claim payments}

We assume that an infectious disease insurance plan collects premiums in the form of continuous annuities from the susceptibles. In other words, policy-holders are committed to paying premiums continuously as long as they remain healthy and susceptible. Meanwhile, medical expenses are continuously reimbursed for each infected policy-holder during the whole period of treatment. Once the individual dies from the disease, the plan terminates immediately.

Following the principles of International Actuarial Notation, we denote the actuarial present value (APV) of premium payments from an insured person for a $t$-year period by $\bar{a}_{t \mid}^{s}$ with the superscript indicating payments from class $S$. The APV of benefits paid by the insurer to the infected at the rate of one monetary unit per time unit is denoted by $\bar{a}_{t}^{i}$ with the superscript indicating payments to class $I$.

We shall use the current payment technique to evaluate the annuities. In other words, we identify the present value of payments due at time $t$, which is the discounted value of one monetary unit for a basic annuity, multiply by the probability of making such a payment and then integrate these actuarial present value for all payment times $t$. A detailed account of evaluations of annuities can be found in Bowers et al. (1997). 
Hence, on the insurance liability side, the total discounted value of a $t$-year annuity of benefit payments is given by

$$
\bar{a}_{\bar{t}}^{i} \triangleq \int_{0}^{t} e^{-\delta x} i(x) d x
$$

where $\delta>0$ is the discounting force of interest. While on the revenue side, the total discounted value of a $t$-year annuity premium of payments is

$$
\bar{a}_{\bar{t}}^{s} \triangleq \int_{0}^{t} e^{-\delta x} s(x) d x
$$

Our study is based on the fundamental notion of the Equivalence Principle for the determination of level premiums, which requires that

$$
\mathbb{E}[\text { present value of benefits }]=\mathbb{E}[\text { present value of benefit premiums }] \text {. }
$$

Therefore, the level premium for a unit annuity claim payment plan is determined by

$$
\bar{P}\left(\bar{a}_{\bar{t}}^{i}\right) \triangleq \frac{\bar{a}_{\bar{t}}^{i}}{\bar{a}_{\bar{t}}^{s}}
$$

As in life insurance, where the force of mortality is defined as the additive inverse of the ratio of the derivative of the survival function to the survival function itself, we define here the force of infection (leaving class $S$ ) as

$$
\mu_{t}^{s} \triangleq-\frac{s^{\prime}(t)}{s(t)}, \quad t \geq 0
$$

and the force of removal (leaving class $I$ ) as

$$
\mu_{t}^{i} \triangleq-\frac{i^{\prime}(t)}{i(t)}, \quad t \geq 0 .
$$

Consequently from (3.1)-(3.2), we see that $\mu_{t}^{s}=\beta i(t)$ and $\mu_{t}^{i}=-\beta s(t)+\alpha$.

Note that the above definitions imply that

$$
s(t)=\exp \left\{-\int_{0}^{t} \mu_{x}^{s} d x\right\}=\exp \left\{-\beta \int_{0}^{t} i(x) d x\right\}, \quad t \geq 0,
$$

and

$$
i(t)=\exp \left\{-\int_{0}^{t} \mu_{x}^{i} d x\right\}=\exp \left\{\beta \int_{0}^{t} s(x) d x+\alpha t\right\}, \quad t \geq 0 .
$$

For mathematical convenience, we shall first analyze the policy with an infinite term. When the policy term is relatively long, the premium based on an infinite term may serve as a rough estimation of the cost of the insurance. 
Proposition 3.1. In the SIR model in (3.1)-(3.2),

$$
\left(1+\frac{\alpha}{\delta}\right) \bar{a}_{\infty}^{i}+\bar{a}_{\bar{\infty}}^{s}=\frac{1}{\delta} .
$$

Note that the right hand side of (3.9) represents the present value of a unit perpetual annuity. The intuitive interpretation of the left hand side is that, if each insured in the whole insured population is provided with a unit perpetual annuity, the APV of payments to class $S$ is given by $\bar{a}_{\bar{\infty}}^{s}$ and the APV of payments to class $I$ is given by $\bar{a}_{\infty}^{i}$. Recall that at any time a fraction $\alpha$ of the infected moves to class $R$. To be fair, each one in class $R$ is also

entitled to a perpetuity worth a value of $1 / \delta$ at the time of transition. Hence the APV of payments to the group leaving class $I$ is given by $(\alpha / \delta) \bar{a}_{\infty}^{i}$. It is reasonable that all three types of annuity payments should add up to the present value of a unit perpetual annuity, $1 / \delta$, paid regardless of which compartment a policy-holder lies in.

From relation (3.9), we easily find the net level premium in (3.6) for a policy of an infinite term with both premium and claim annuity payments given by the formula

$$
\bar{P}\left(\bar{a}_{\infty 1}^{i}\right)=\frac{\bar{a}_{\bar{\infty}}^{i}}{\bar{a}_{\infty}^{s}}=\frac{\delta \bar{a}_{\infty}^{i}}{1-(\delta+\alpha) \bar{a}_{\bar{\infty}}^{i}} .
$$

\subsection{Annuity premium and lump sum claim payments}

An insurance plan that pays a lump sum compensation would be analogous to a whole life insurance in actuarial mathematics. When an insured person is diagnosed with the infectious disease and immediately hospitalized, the medical expenses are to be paid immediately in a lump sum and the insurance plan terminates as its obligation is fulfilled. Then the APV of benefit payments, denoted by $\bar{A}_{\infty}^{i}$, is given by

$$
\bar{A}_{\infty}^{i} \triangleq \beta \int_{0}^{\infty} e^{-\delta t} s(t) i(t) d t,
$$

since the probability of being newly infected at time $t$ is $\beta s(t) i(t)$.

Proposition 3.2. In the SIR model in (3.1)-(3.2),

$$
\frac{1}{\delta} \bar{A}_{\infty\rceil}^{i}+\bar{a}_{\bar{\infty}}^{s}=\frac{1}{\delta} s_{0},
$$

and

$$
\frac{1}{\delta} i_{0}+\frac{1}{\delta} \bar{A}_{\infty \mathbf{}}^{i}=\frac{\alpha}{\delta} \bar{a}_{\infty \mid}^{i}+\bar{a}_{\infty \downarrow}^{i} .
$$


Equation (3.12) also provides insight into the break-down of expenses in each class. Assuming that every susceptible individual who initially enters the policy claims a unit perpetual annuity, then APV of the total cost is $s_{0} / \delta$. From a different perspective, it is equivalent to give every one a unit of annuity as long as the insured remains healthy in the group and then grant each a unit perpetuity immediately as they become infected. The APV of these two types of payments is exactly given by $(1 / \delta) \bar{A}_{\infty}^{i}+\bar{a}_{\infty}^{s}$.

If one thinks of class $I$ as a transit stage, then we can count the costs of payments on both the incoming and outgoing sources. Assume that every one currently or previously in class $I$ receives a unit of perpetuity. From the incoming sources, the left hand side of (3.13) gives the expenses for initial members $i_{0} / \delta$ and the expenses for those who just entered the class $(1 / \delta) \bar{A}_{\infty}^{i}$. For the outgoing sources, the costs for individuals who continue to stay in the class is given by $\bar{a}_{\infty}^{i}$ and those deceased are compensated with a perpetuity worth $1 / \delta$. Thus the right hand side sums up to $(\alpha / \delta) \bar{a}_{\infty \mid}^{i}+\bar{a}_{\bar{\infty} \text {. }}^{i}$

Therefore the net level premium $\bar{P}\left(\bar{A}_{\infty}^{i}\right)$ for the plan of an infinite term insurance with lump sum compensation and annuity premium payments is given by the equivalence principle:

$$
\bar{P}\left(\bar{A}_{\infty}^{i}\right) \triangleq \frac{\bar{A}_{\bar{\infty}}^{i}}{\bar{a}_{\infty}^{s}}=\frac{(\alpha+\delta) \bar{a}_{\bar{\infty}}^{i}-i_{0}}{1-(\alpha+\delta) \bar{a}_{\infty}^{i}} .
$$

\subsection{Death benefit}

Note that in the epidemiological literature the class $R$ is composed of all individuals removed chronologically from a previous compartment, who either recover with immunity or die from the disease. A more refined model would have separate compartments for deaths and recovered individuals. For our purpose of investigating actuarial implications of the epidemiological model, we keep the simple assumption of only one $R$ compartment exclusively for deaths caused by the disease.

Health insurance plans often have death benefits that differ in value from health-care benefits. In this infectious disease plan, we assume a death benefit of a monetary unit paid immediately at the moment of death. Thus, the APV of a lump sum death benefit payment, denoted by $\bar{A}_{\infty}^{d}$, is given by

$$
\bar{A}_{\infty\rceil}^{d} \triangleq \alpha \int_{0}^{\infty} e^{-\delta t} i(t) d t=\alpha \bar{a}_{\infty\rceil}^{i} .
$$

Therefore, the net level premium for the plan of an infinite term with both a unit lump sum death benefit and health-care claim is obtained by:

$$
\bar{P}\left(\bar{a}_{\infty \mid}^{i}+\bar{A}_{\infty \mid}^{d}\right) \triangleq \frac{\bar{a}_{\infty}^{i}+\bar{A}_{\infty}^{d}}{\bar{a}_{\infty}^{s}}=\frac{\delta(1+\alpha) \bar{a}_{\infty}^{i}}{1-(\alpha+\delta) \bar{a}_{\infty}^{i}} .
$$


Finally, the net level premium for a plan with both, a lump sum benefit for hospitalization costs and a lump sum death benefit is given by:

$$
\bar{P}\left(\bar{A}_{\infty \mid}^{i}+\bar{A}_{\bar{\infty}}^{d}\right) \triangleq \frac{\bar{A}_{\bar{\infty}}^{i}+\bar{A}_{\bar{\infty}}^{d}}{\bar{a}_{\infty}^{s}}=\frac{(\delta+\alpha+\delta \alpha) \bar{a}_{\bar{\infty}}^{i}-i_{0}}{1-(\alpha+\delta) \bar{a}_{\bar{\infty}}^{i}} .
$$

\section{Ratemaking}

So far net premiums have only been expressed in terms of $\bar{a}_{\infty}^{i}$, which is a Laplace transform of $i(t)$. An implicit integral solution to the SIR model in (3.1)-(3.2) is as follows,

$$
\begin{aligned}
s(t) & =\frac{1}{N} \exp \left\{-\beta \int_{0}^{t} \exp \left\{\beta N \int_{0}^{u} s(x) d x-\alpha u\right\} d u\right\}, & t \geq 0, \\
i(t) & =\frac{1}{N} \exp \left\{\beta \int_{0}^{t} \exp \left\{\beta N \int_{0}^{u} i(x) d x\right\}-\alpha u d u\right\}, & t \geq 0 .
\end{aligned}
$$

No general explicit solution is available for $s(t)$ and $i(t)$. Therefore we propose numerical methods and approximations that can provide satisfactory solutions for insurance applications. The estimation of $i(t)$ enables us to compute $\bar{a}_{\bar{\infty}}^{i}$, which in turn gives $\bar{a}_{\bar{\infty}}^{s}$ via the relation between $\bar{a}_{\bar{\infty}}^{i}$ and $\bar{a}_{\bar{\infty}}^{s}$.

In addition the proposed techniques are extended to the more realistic finite term policy. These numerical methods generally apply to the calculations of both the infinite and finite term policy.

\subsection{Infection table based approximation}

In practice it is difficult to keep record of susceptible individuals, partly because of their large numbers in a population and partly due to the difficulty in distinguishing a person susceptible to a certain disease from one with immunity. But we can keep track of infected people using public data from government health agencies and hospitals. Hence we rely on the function $i(t)$, instead of $s(t)$, for all premium rating calculations.

A natural analogy here is with the life table in life insurance mathematics, which virtually describes an empirical survival distribution of an average person's longevity. Similarly, an infection table can be generated to keep record of the number of infected cases reported during each sampling period (e.g., every day for SARS). Table 2 in Section 6.1 is a simple example of an infection table dated back to the 17th century.

Now from the infection table, we have a piecewise constant empirical approximation of the continuous function $i(t)$ given by

$$
\tilde{\imath}(t)= \begin{cases}i_{k}, & k-1<t \leq k \\ 0, & \text { otherwise }\end{cases}
$$


where $i_{k}$ is the rate of infection in the $k$-th period on the infection table.

Using this function in place of $i(t)$ in (3.4) gives an approximation to $\bar{a}_{\bar{t}}^{i}$,

$$
\bar{a}_{\bar{t}}^{i}=\int_{0}^{t} e^{-\delta x} i(x) d x \approx \int_{0}^{t} e^{-\delta x} \tilde{\imath}(x) d x \approx \sum_{k=1}^{n} \frac{e^{-\delta(k-1)}-e^{-\delta k}}{\delta} i_{k},
$$

where $n=[t]$ is the integer part of $t$, and $n$ is large enough. To compute a perpetuity $\bar{a}_{\bar{\infty}}^{i}$, one needs to choose a sufficiently large term $n$ and hence find $\bar{P}\left(\bar{a}_{\infty}^{i}\right)$ by (3.10) as an approximate asymptotic values.

Following the same logic as in Proposition 3.1, the following relation between $\bar{a}_{\bar{t}}^{s}$ and $\bar{a}_{\bar{t}}^{i}$ is easily obtained:

$$
\bar{a}_{\bar{t}}^{s}=\frac{1}{\delta}\left(1-e^{-\delta t}\right)+\frac{\alpha}{\delta} e^{-\delta t} \int_{0}^{t} i(r) d r-\left(\frac{\alpha}{\delta}+1\right) \bar{a}_{\bar{t}}^{i}
$$

Therefore, the premium for the policy with annuity claims and payments can be calculated by

$$
\pi_{t}=\frac{\bar{a}_{\bar{t} \mid}^{i}}{\bar{a}_{\bar{t}}^{s}} \approx \frac{(1 / \delta) \sum_{k=1}^{n}\left[e^{-\delta(k-1)}-e^{-\delta k}\right] i_{k}}{(1 / \delta)\left(1-e^{-\delta t}+(\alpha / \delta) e^{-\delta t} \sum_{k=1}^{n} i_{k}-\left(\alpha / \delta^{2}+1 / \delta\right) \sum_{k=1}^{n}\left[e^{-\delta(k-1)}-e^{-\delta k}\right] i_{k}\right.} .
$$

\subsection{Power series solutions}

The power series method is one of the oldest techniques used to solve linear differential equations. This method can be adapted well to our SIR model.

Since every point in the system is an ordinary point, in particular, $t=0$, we look for solutions of the form

$$
\begin{array}{ll}
s(t)=\sum_{n=0}^{\infty} a_{n} t^{n}, & t \geq 0, \\
i(t)=\sum_{n=0}^{\infty} b_{n} t^{n}, & t \geq 0 .
\end{array}
$$

Therefore, differentiating term by term yields

$$
\begin{array}{ll}
s^{\prime}(t)=\sum_{n=1}^{\infty} n a_{n} t^{n-1}=\sum_{n=0}^{\infty}(n+1) a_{n+1} t^{n}, \quad t \geq 0, \\
i^{\prime}(t)=\sum_{n=1}^{\infty} n b_{n} t^{n-1}=\sum_{n=0}^{\infty}(n+1) b_{n+1} t^{n}, \quad t \geq 0 .
\end{array}
$$

Multiplying (4.1) by (4.2) gives,

$$
s(t) i(t)=\sum_{n=0}^{\infty} c_{n} t^{n}, \quad t \geq 0
$$


where

$$
c_{n}=a_{0} b_{n}+a_{1} b_{n-1}+\cdots+a_{n-1} b_{1}+a_{n} b_{0} .
$$

From (3.1), we obtain

$$
\begin{gathered}
\sum_{n=0}^{\infty}(n+1) a_{n+1} t^{n}+\beta \sum_{n=0}^{\infty} c_{n} t^{n}=0, \\
\sum_{n=0}^{\infty}(n+1) b_{n+1} t^{n}-\beta \sum_{n=0}^{\infty} c_{n} t^{n}+\alpha \sum_{n=0}^{\infty} b_{n} t^{n}=0 .
\end{gathered}
$$

To satisfy these equations for all $t$, it is necessary that the coefficient of each power of $t$ be zero. Hence we obtain the following recursion relation:

$$
\begin{gathered}
a_{n+1}=-\frac{\beta}{n+1}\left(a_{0} b_{n}+a_{1} b_{n-1}+\cdots+a_{n-1} b_{1}+a_{n} b_{0}\right), \\
b_{n+1}=-a_{n+1}-\frac{\alpha}{n+1} b_{n} .
\end{gathered}
$$

Therefore,

$$
\begin{aligned}
\bar{a}_{\bar{t}}^{i} & =\sum_{n=0}^{\infty} \int_{0}^{t} b_{n} e^{-\delta x} x^{n} d x=\sum_{n=0}^{\infty} b_{n} \Gamma(n+1) \Gamma(n+1, t), \\
\bar{a}_{\bar{t}}^{s}=\sum_{n=0}^{\infty} \int_{0}^{t} a_{n} e^{-\delta x} x^{n} d x & =\sum_{n=0}^{\infty} a_{n} \Gamma(n+1) \Gamma(n+1, t),
\end{aligned}
$$

where $\Gamma(n, t)$ is the incomplete gamma function

$$
\Gamma(n, t)=\frac{1}{\Gamma(n)} \int_{0}^{t} x^{n-1} e^{-t} d t, \quad n>0, t>0 .
$$

which is readily available numerically in most mathematical or statistical software.

Hence the premium for the policy with annuity claims and payments can be calculated by

$$
\pi_{t}=\frac{\bar{a}_{\hat{t}}^{i}}{\bar{a}_{t}^{s}}=\frac{\sum_{n=0}^{\infty} b_{n} \Gamma(n+1) \Gamma(n+1, t)}{\sum_{n=0}^{\infty} a_{n} \Gamma(n+1) \Gamma(n+1, t)} .
$$

Interestingly, when $t \rightarrow \infty$, these formulas simplify to

$$
\bar{a}_{\infty \mid}^{i}=\sum_{n=0}^{\infty}(n !) b_{n} \quad \text { and } \quad \bar{a}_{\varnothing \mid}^{s}=\sum_{n=0}^{\infty}(n !) a_{n},
$$

which implies that

$$
\bar{P}\left(\bar{a}_{\infty}^{i}\right)=\frac{\sum_{n=0}^{\infty}(n !) b_{n}}{\sum_{n=0}^{\infty}(n !) a_{n}} .
$$




\subsection{Insurance related quantities and Runge-Kutta method}

Among many numerical methods for solving ODE's, the Runge-Kutta method is the most popular. It can be adapted for any order of accuracy. For applications in insurance, the fourth order Runge-Kutta method (RK-4), given by the following recursion formulas, represents a good compromise between simplicity and accuracy:

$$
\begin{aligned}
y_{i+1} & =y_{i}+\frac{1}{6}\left(k_{1 i}+2 k_{2 i}+3 k_{3 i}+k_{4 i}\right), \quad i=1,2, \ldots, n, \\
k_{1 i} & =h f\left(t_{i}, y_{i}\right), \quad k_{2 i}=h f\left(t_{i}+\frac{h}{2}, y_{i}+\frac{1}{2} k_{1 i}\right), \\
k_{3 i} & =h f\left(t_{i}+\frac{h}{2}, y_{i}+\frac{1}{2} k_{2 i}\right), \quad k_{4 i}=h f\left(t_{i}+h, y_{i}+k_{3 i}\right),
\end{aligned}
$$

where $y_{i}$ is given by the ODE:

$$
\frac{d y}{d t}=f(t, y),
$$

evaluated at $t=t_{i}$, and where $h=t_{i}-t_{i-1}$ is the time step, for $i=1,2, \ldots, n$. The Runge-Kutta method is discussed in detail by Boyce and DiPrima (1986).

Actuaries will be interested particularly in the properties of insurance-related quantities, such as the total discounted benefits, the total discounted premiums and the premium reserves. Based on the RK-4 method, we need to express these quantities into a system of differential equations. Let $P(t)$ denote the accumulated value of premiums collected up to time $t$ and $B(t)$ the corresponding accumulated value of benefits paid up to time $t$. Using a retrospective approach, we consider $V(t)$, the accumulated benefit reserve at time $t$, as the difference between the accumulated value of premiums and the accumulated value of claims.

Assume that the infectious disease plan with annuity premium payments provides one monetary unit of compensation per time unit for infected policy-holders. Then the connections among these insurance-related and epidemiological quantities could be described by the following ODE system:

$$
\begin{aligned}
P^{\prime}(t) & =\pi s(t)+\delta P(t), & t>0, \\
B^{\prime}(t) & =i(t)+\delta B(t), & t>0, \\
V(t) & =P(t)-B(t), & t>0,
\end{aligned}
$$

where $P(0)=\pi s_{0}, B(0)=i_{0}$ and $p$ is a testing premium rate. The rationale behind the ODE is as follows. The instantaneous change in the accumulated value of total premiums $P^{\prime}(t)$ is given by the sum of the instantaneous rate of premium income $\pi s^{\prime}(t)$ and the instantaneous rate of interest return on the current total premiums $\delta P(t)$. The instantaneous change in the accumulated value of total claims is the sum of the instantaneous rate of claims $i^{\prime}(t)$ and the instantaneous rate of interest return on the current total claims $\delta S(t)$. 
These ODE systems can be readily solved in most mathematical software such as Maple. Information about programming with the ODE tool kits in Maple can be found in Barnes and Fulford (2008) and Coombes et al. (1997).

We first use $\pi=\bar{P}\left(\bar{A}_{\infty}^{i}\right)$ as a starting point to test the behaviour of the reserve function. Then we can gradually increase the premium rate $\pi$ in order to produce an acceptable reserve schedule.

\section{Premium adjustments}

We shall first investigate the demographic changes in the insured group over time.

Proposition 5.1. For the SIR model in (3.1)-(3.3), $s(t)$ is monotonically decreasing in $t$, and $r(t)$ is monotonically increasing. If $s_{0} \leq \alpha / \beta$, then $i(t)$ is monotonically decreasing, while if $s_{0}>\alpha / \beta, i(t)$ increases up to the time $t^{*}$ at which point $s\left(t^{*}\right)=\alpha / \beta$, and then decreases afterwards.

In actuarial mathematics mortality rates mostly rise with age. If premiums are held constant, the insurer's future liability exceeds the future premium revenue. To cover future liabilities, life insurers set aside benefit reserves. Unlike the "U" shape of mortality curves, a unique feature of epidemics is that the infection rates increase rapidly at the beginning but then drop after reaching a peak, as proved in Proposition 5.1. Figure 4 illustrates a typical path of a benefit reserve function obtained for the policy in (4.3)-(4.5), where the benefit premium is determined by (3.6). As one can see, because infection rates increase rapidly in the early stage and drop significantly at the later stage, an insurer's liability is larger at the beginning but decreases over the time. Level premiums over a long term imply negative benefit reserves, as claims exceed premiums in the early stages of the epidemic, as well as the reserve at the later stage. Since there is a likelihood of policy withdrawal after the peak of infection, an insurer may not be able to collect enough premiums. Hence the need to increase the premium to a level that guarantees positive benefit reserve even if this means that the insurer will have to pay a cash-value to policy-holders at the term of the policy.

We shall now investigate how the premium rate $\pi$ affects the shape of benefit reserve function using the example of a plan with both, annuity premiums and claim payments.

It follows immediately from (4.3)-(4.5) that

$$
V^{\prime}(t)=\pi s(t)-i(t)+\delta V(t), \quad t \geq 0 .
$$

For simplicity, consider the case where the force of interest $\delta=0$, in order to better grasp the connection between $\pi$ and the shape of the benefit reserve. We leave the more complicated case $\delta>0$ for the numerical analysis at the end of this section. 
Since the sign of the instantaneous change in $V(t)$ depends on two competing forces, the monotonically decreasing $\pi s(t)$ and the decreasing or increasing-then-decreasing $i(t)$. There are four possible shapes of the graph of the benefit reserve $V(t)$, namely, strictly increasing concave, strictly increasing concave-then-convex, non-monotonic concave-then-convex and non-monotonic convex, as shown in Figures 2-5, respectively. The following propositions provide conditions under which the four scenarios appear (see the Appendix for the proofs).

Proposition 5.2. (Convexity) In (4.3)-(4.5) with $\delta=0$, the shape of benefit reserve $V(t)$ is determined by the premium rate $\pi$ as follows:

1. $V(t)$ is concave, if

$$
\pi \geq \frac{\alpha}{\beta s_{\infty}}-1
$$

where the constant $s_{\infty}=\lim _{t \rightarrow \infty} s(t)$.

2. $V(t)$ changes from concave to convex, if

$$
\frac{\alpha}{\beta s_{0}}-1<\pi<\frac{\alpha}{\beta s_{\infty}}-1
$$

The point of inflection $t_{f}$ is given by

$$
s\left(t_{f}\right)=\frac{\alpha}{(1+\pi) \beta} .
$$

3. $V(t)$ is convex, if

$$
\pi \leq \frac{\alpha}{\beta s_{0}}-1
$$

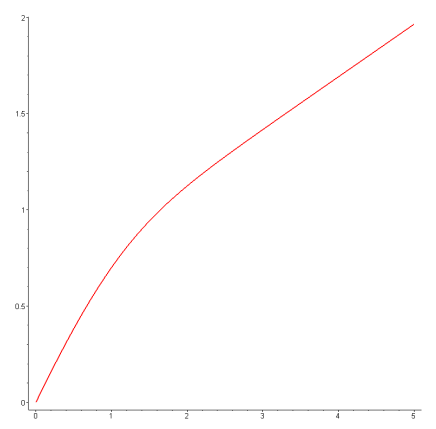

Figure 2: Benefit reserve function $V(t)$ of the Great Plague plan with $\pi=0.86$ 


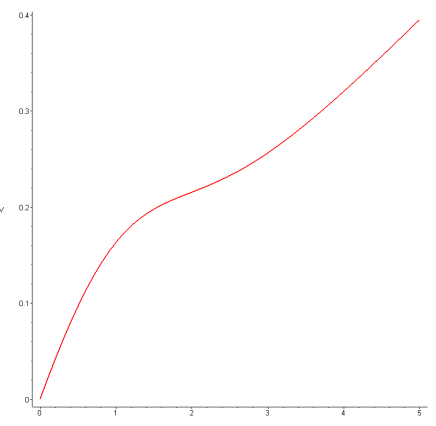

Figure 3: Benefit reserve function $V(t)$ of the Great Plague plan with $\pi=0.25$.

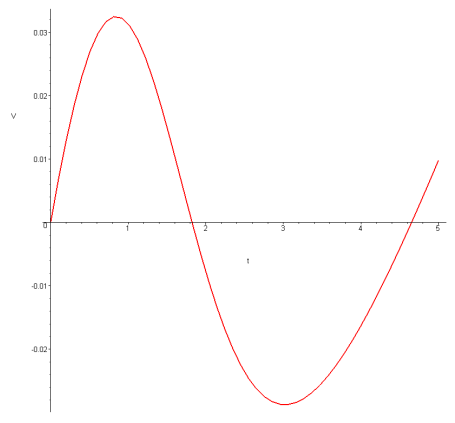

Figure 4: Benefit reserve function $V(t)$ of the Great Plague plan with $\pi=0.10$.

Proposition 5.3. (Monotonicity) In (4.3)-(4.5), the reserve $V(t)$ is strictly increasing, if

$$
\pi>\frac{\alpha}{\beta} \exp \left(\frac{\beta c}{\alpha}-1\right)-1,
$$

where the constant $c=1-\alpha \ln \left(s_{0}\right) / \beta$.

Since $s(t)$ is a decreasing function, it is easy to see that

$$
\frac{\alpha}{\beta s_{\infty}}-1 \leq \frac{\alpha}{\beta} \exp \left\{\frac{\beta c}{\alpha}-1\right\}-1 \leq \frac{\alpha}{\beta s_{0}}-1 .
$$

Table 1 summarizes the four possible shapes that result for reserve functions.

When $\delta$ is small relative to $\beta i(r)$, for $r \in[0, t)$, the shape of the reserve function does not change significantly. Hence we can use the relation between $\pi$ and $V(t)$, when $\delta=0$, as our starting approximation to search for an accurate premium in the case when $\delta>0$.

As we can see from Figures 4 and 5, premium rates are quite low but there might be undesirable negative reserves during the policy term. On the other hand the insurer may not favour a policy design with the strictly increasing liability shown in Figure 3, as premiums are relatively high, increasing moral hazard. It means that healthy policy-holders are more 


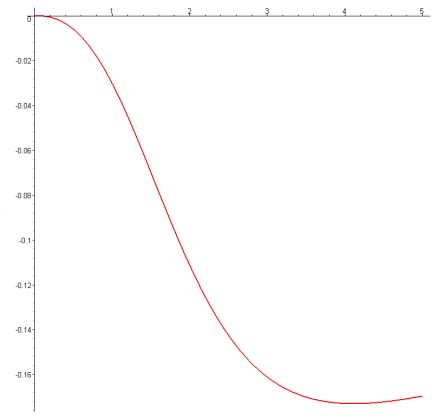

Figure 5: Benefit reserve function $V(t)$ of the Great Plague plan with $\pi=0.03$

\begin{tabular}{|l|l|}
\hline Shape of $V(t)$ & Interval for Values of $\pi$ \\
\hline Increasing concave & {$\left[\alpha /\left(\beta s_{0}\right)-1, \infty\right)$} \\
\hline Increasing concave-then-convex & {$\left[\alpha / \beta \exp \{\beta c / \alpha-1\}-1, \alpha /\left(\beta s_{0}\right)-1\right)$} \\
\hline Non-monotonic concave-then-convex & {$\left[\alpha /\left(\beta s_{\infty}\right)-1, \alpha / \beta \exp \{\beta c / \alpha-1\}-1\right)$} \\
\hline Non-monotonic convex & {$\left[0, \alpha /\left(\beta s_{\infty}\right)-1\right)$} \\
\hline
\end{tabular}

Table 1: Possible Shapes of $V(t)$ when $\delta=0$

likely to shop for lower premium rates while unhealthy ones keep the policy, ultimately increasing insurance costs. Hence the need for a design with more marketable premiums that produce a bell--shaped reserve, that is a concave-then-convex shape, with the relatively low final cash-values shown in Figure 7.

The above analysis shows the importance of determining whether the reserve function is concave at the term of the policy. If so, premiums can gradually increase so that the reserve reaches zero at the term of the policy, producing a bell-shaped reserve. Otherwise, a premium that produces a bell-shaped reserve might not exist, hence the need to settle down for a concave-then-convex reserve function, producing a positive cash-value paid out at the end of the policy term.

The following algorithm calculates a premium rate for a $t$-year policy with non-negative cash-values over the whole policy term. The main idea of the algorithm is summarized as follows: It starts with an initial premium rate that ensures that $V(t)$ is in its concave phase at the end of the policy if $\delta=0$. Since a small $\delta$ will not affect the shape of $V(t)$ in a significant way, this step gives a tractable initial premium that can be used for other $\delta$ values. As Step 1 is part of a loop, it is later used to check whether $V(t)$ is still in a concave phase with the newly adjusted premium rate. If so, proceed to Step 2.1 which adjusts premium rates according to the sign of $V(t)$. If a premium with zero final cash-value is feasible, it will be obtained in Step 2.1. Step 2.2 deals with the case when $V(t)$ is in a convex phase. 
If $V(t)<0$, it means that the reserve has not yet reached its lowest level at the policy term. Hence it is necessary to increase the premium until $V(t) \approx 0$. If $V(t)$ increases from negative to positive in one step, it simply means that a policy with zero final cash-value is not feasible, but the premium is as low as possible with non-negative reserves.

- Step 0: Set $n:=1$ and $\pi^{(1)}$ is set to be relatively small such that $\alpha /(\beta s(t))-1<$ $\pi^{(1)}<\alpha /\left(\beta s_{\infty}\right)-1$.

- Step 1: If $\pi^{(n)}>[\beta i(t) s(t)+\delta i(t)-\alpha i(t)] /[\delta s(t)-\beta s(t) i(t)]$, proceed to Step 2.1. Otherwise, proceed to Step 2.2.

- Step 2.1: If $V(t)<0$, then $\pi^{(n+1)}:=\pi^{(n)}+0.01$, set $n:=n+1$ and go to Step 1. If $V(t)>0$, then $\pi^{(n+1)}:=\pi^{(n)}-0.01$, set $n:=n+1$ and go to Step 1 . If $V(t) \approx 0$, stop and $\pi^{(n)}$ is the premium rate with zero final cash-value.

- Step 2.2: If $V(t)<0$, then $\pi^{(n+1)}:=\pi^{(n)}+0.01$, set $n:=n+1$ and go to Step 2.2. If $V(t) \approx 0$, stop and $\pi^{(n)}$ is the premium rate with zero final cash-value. If $V(t)>0$, stop and $\pi^{(n)}$ is the smallest premium rate with positive final cash-value $V(t)$ at the end of the policy.

If the algorithm produces a positive value of $V(t)$, it implies that the reserve function reaches its lowest point, a non-negative value, prior to the end of the policy. By the Equivalence Principle, the cash-value $V(t)$ should be paid back to all surviving policy-holders at the policy term.

\section{$6 \quad$ Numerical examples}

The epidemiological model in our first numerical example of the Great Plague in Eyam was originally studied by Raggett (1982). It is included as a classical case study in many textbooks because predictions from the model are remarkably close to actual data. The second example of a six compartment model comes from Chowell et al. (2003), where parameters are primarily used for measuring the mean numbers of secondary cases a single infected will cause in a population with no immunity, in the absence of interventions to control the infection. However, our focus is on the insurance arrangement to alleviate the financial burden on the population, rather than model fitting and selection. These two datasets and compartment models are assumed to give an accurate depiction of the dynamics of corresponding diseases upon which actuarial models are built. 


\subsection{Great Plague in Eyam}

The village of Eyam near Sheffield, England, suffered a severe outbreak of bubonic plague in 1665-1666. The plague was survived by only 83 of an initial population of 350 villagers. Detailed records were preserved as shown, in Table 2. In Raggett (1982), the SIR model is fitted to the Eyam data, over the period from mid-May to mid-October 1666. Time is measured in months, with an initial population of 7 infectives and 254 susceptibles, and a final population of 83 . Since the disease was fatal at the time, all infected individuals eventually died from to the disease.

\begin{tabular}{|c|c|c|}
\hline Date & Susceptibles & Infectives \\
\hline Initial & 254 & 7 \\
\hline July 3-4 & 235 & 14.5 \\
\hline July 19 & 201 & 22 \\
\hline August 3-4 & 153.5 & 29 \\
\hline August 19 & 121 & 21 \\
\hline September 3-4 & 108 & 8 \\
\hline September 19 & 97 & 8 \\
\hline October 4-5 & Unknown & Unknown \\
\hline October 20 & 83 & 0 \\
\hline
\end{tabular}

Table 2: Eyam plague susceptible and infective populations in 1666. Data source: Raggett (1982), Table II.

In order to set up an actuarial model based on the arrangement proposed in Figure 1, we shall base the actuarial analysis on an accurately calibrated SIR model.

According to (7.5),

$$
i_{0}+s_{0}-\frac{\alpha}{\beta} \ln s_{0}=i_{\infty}+s_{\infty}-\frac{\alpha}{\beta} \ln s_{\infty},
$$

from which we obtain an expression for $\beta / \alpha$ in terms of measurable quantities $s_{0}$ and $s_{\infty}$,

$$
\frac{\beta}{\alpha} \approx \frac{\ln \left(s_{0} / s_{\infty}\right)}{1-s_{\infty}} .
$$

It is generally difficult to estimate the contact rate $\beta$ due to its dependency on social and behavioural factors. Hence it is important that we can estimate $\beta$ from (6.1) with an estimation of the fatality rate $\alpha$.

Since $s_{0}=254 / 261=0.97318, i_{0}=7 / 261=0.02682$ and $s_{\infty}=83 / 261=0.31801$, the equation (6.1) gives $\beta / \alpha=1.64004$. The parameter $\alpha$ is determined by its reciprocal, which 

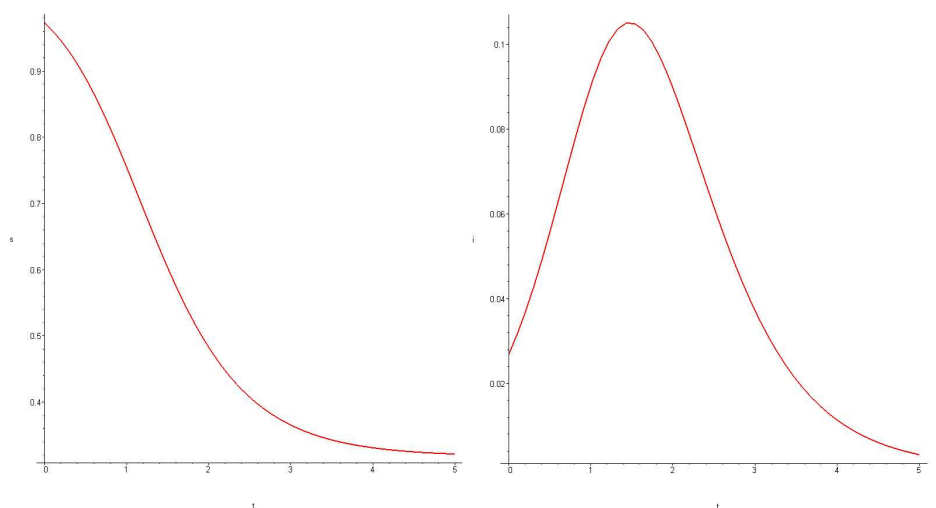

Figure 6: Percentage of the susceptibles $s(t)$ and percentage of the infected $i(t)$

has the clinical interpretation of the average infectious period. From clinical observations, an infected person stays infectious for an average of 11 days or 0.3667 months before death, so that $\alpha=1 / 0.3667=2.73$ and $\beta=4.4773$. The resulting graphs of $s(t)$ and $i(t)$ are given in Figure 6.

Insurance coverage will not directly reduce the transmission of the disease, if an epidemic similar to that described in Table were to occur today. But a well-designed insurance program could provide financial incentives for prevention measures and compensations for hospitalization and other medical costs/services. To develop this insurance model, we assume that everyone in the village participates in a mutual insurance fund set up at the beginning of the epidemic. The insurance fund earns interest at the force of interest of $0.2 \%$. The insurance term lasts 5 months, which matches the duration of the epidemic.

The insurance plan provides infection benefits continuously at the rate of $\$ 1,000$ per month until death, for every infected individual, for the whole period when he or she has been infected and hospitalized. The insurance liability is terminated after death. It is purchased by susceptible individuals, through continuous premium payments.

Using the algorithm provided in Section 5, we start with an initial premium rate $\pi^{(1)}=$ $1000 \alpha /[\beta s(5)]-1=917.37$. The initial premium is quite high because the algorithm requires the reserve to stay in its concave phase at the start, to then gradually reduce the premium to a desired level. After many rounds of premium reduction, we reach the final premium rate of $\pi=114.58$. Such a premium rate avoids the reserve from ever being negative and keeps the cash-value at policy end at a reasonable level of $V(5)=49.44$, which means each survivor receives a reward of $\$ 49.44$ at the end of the epidemic to clear off the insurance fund. The reserve function of such a policy is given in Figure 7. 


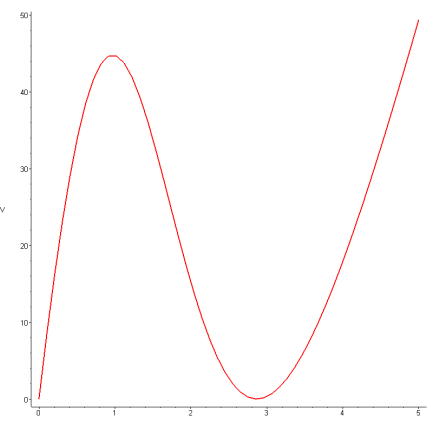

Figure 7: Cash-values of the Great Plague plan

\subsection{SARS epidemic in Hong Kong}

In the classical SIR model, the implicit assumption that the mixing of members from different compartments is geographically homogeneous is probably unrealistic. The susceptible people in geographical neighborhoods of an infectious virus-carrier are more likely to be infected than those who are remote from the carrier. For instance, during the SARS epidemic in Hong Kong, it was observed that health care workers were at higher risk of infection than most other groups in the population.

To distinguish different levels of vulnerability or infectiousness within different social groups, spatial structures are introduced and developed in epidemiological studies. A typical example of a spatial structure applied to the SARS epidemic in Hong Kong is defined by Chowell et al. (2003) in the following ODE system,

$$
\begin{aligned}
S_{1}^{\prime}(t) & =-\beta S_{1}(t)\left[\frac{I(t)+q E(t)+l J(t)}{N}\right], \quad t \geq 0, \\
S_{2}^{\prime}(t) & =-\beta p S_{2}(t)\left[\frac{I(t)+q E(t)+l J(t)}{N}\right], \quad t \geq 0, \\
E^{\prime}(t) & =\beta\left(S_{1}(t)+p S_{2}(t)\right)\left[\frac{I(t)+q E(t)+l J(t)}{N}\right]-k E(t), \quad t \geq 0, \\
I^{\prime}(t) & =k E(t)-\left(\alpha+\gamma_{1}+\tau\right) I(t), \quad t \geq 0, \\
J^{\prime}(t) & =\alpha I(t)-\left(\gamma_{2}+\tau\right) J(t), \quad t \geq 0, \\
R^{\prime}(t) & =\gamma_{1} I(t)+\gamma_{2} J(t), \quad t \geq 0 .
\end{aligned}
$$

In this model, there are two distinct susceptible compartments with different levels of exposure to SARS, namely $S_{1}$ for the most susceptible urban community and $S_{2}$ for the less susceptible rural population. Initially, $S_{1}(0)=\rho N$ and $S_{2}(0)=(1-\rho) N$, where $\rho$ is the proportion of urban individuals in the total population. An average highly susceptible person (in Class $S_{1}$ ) makes an average number of $\beta$ risky contacts (i.e. contacts sufficient to transmit infection) with others per unit time. Because of less frequent visits to public areas 
where viruses concentrate, an average lower susceptible person (in Class $S_{2}$ ) would only be exposed to an average number of $p \beta$ risky contacts with others per unit time.

Since individuals infected with SARS experience incubation periods of $2-7$ days before the onset of any visible symptoms, an infectious class is set up for those infected but not yet symptomatic. The parameter $q$ is used to measure the lower level of infectivity during the incubation. With time, the infected individual develops observable symptoms and becomes fully infectious, in Class $I$ with $q=1$. In order to distinguish their potential disease transmission to the general public, Class $I$ is separated for those infectious individuals still un-diagnosed. Since almost all diagnosed cases are quarantined in hospitals, Class $J$ has a lower infectivity level reflected by a reduction factor $l$.

The population rates transferring from $E, I$ and $J$ to their chronologically adjacent compartments $I, J$ and the recovered Class $R$, are respectively $k, \alpha$ and $\gamma_{2}$. Considering that even before being diagnosed, SARS patients may either recover naturally at the rate of $\gamma_{1}$ or die at the force of death $\tau$, we also have Class $D$, tallying deaths as a result of SARS, from sources $I$ and $J$. The patients under medical treatment in Class $J$ suffer death at the rate assumed to be the same as the mortality rate in Class $I$.

Notice that both $E$ and $I$ are un-diagnosed phases, there is literally no statistical data for estimating their parameters. Therefore, another compartment $C$ for reported probable cases is used to trace back, by a time series, the original time of incidence. Figure 8 illustrates the possible transfer vectors among the different compartments.

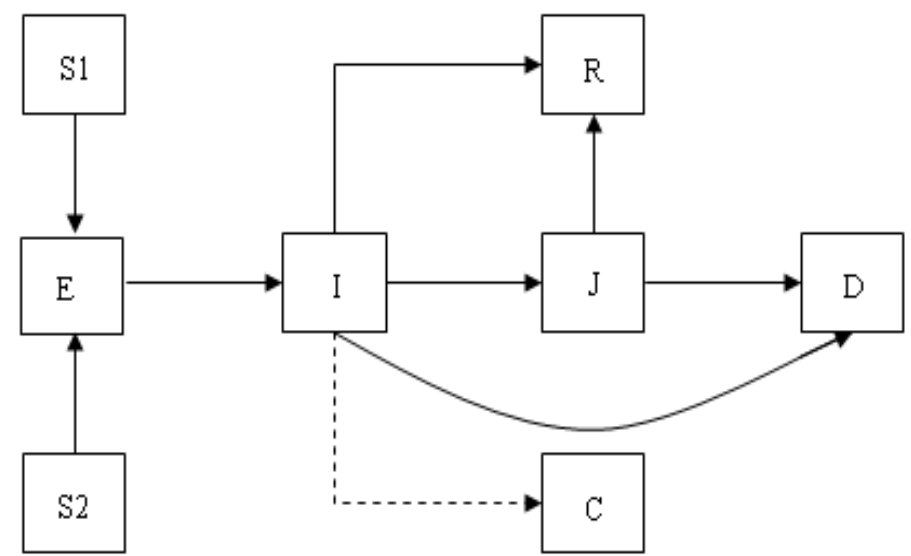

Figure 8: Transfer diagram of the SARS epidemic dynamics (reprint of Figure 1 in Chowell et al. 2003).

Leaving aside the detailed parameter inference analysis, we use the parameter estimates in Chowell et al. (2003), summarized in Table 3. These allow the computation of the basic reproductive number $R_{0}$ in the original article. A drawback of this model is that it can lead 
to some negative counts in Classes $I$ and $J$.

\begin{tabular}{|c|c|c|}
\hline Parameter & Moving from/to & Value \\
\hline$\beta$ & $S_{1}, S_{2} / E$ & 0.75 \\
\hline$q$ & reduced infectiousness & 0.1 \\
\hline$l$ & reduced infectiousness & 0.38 \\
\hline$p$ & reduced susceptibility & 0.1 \\
\hline$k$ & $E / I$ & $1 / 3$ \\
\hline$\alpha$ & $I / J$ & $1 / 3$ \\
\hline$\gamma_{1}$ & $I / R$ & $1 / 8$ \\
\hline$\gamma_{2}$ & $J / R$ & $1 / 5$ \\
\hline$\tau$ & $I, J / R$ & 0.006 \\
\hline$\rho$ & reduced contacts & 0.4 \\
\hline
\end{tabular}

Table 3: Parameter values fitted to the SARS model for Hong Kong (see Table 1 in Chowell et al. 2003).

From an insurer's point of view, this model presents interesting business opportunities. On the one hand, individuals in Classes $S_{1}$ and $S_{2}$ are potential buyers facing the risk of infection with SARS. On the other hand, there is an evident need for insurance covering vaccination costs in both $S_{1}$ and $S_{2}$, medical examination expenses for probable cases in Class $I$, hospitalization and quarantine expenses for Class $J$ and death benefits for Class $D$.

Several parties have stakes in our health care systems, such as insurance companies, policy-holders, government health agencies, and hospitals. Numerous business models could be designed to reduce the overall financial impact to a minimum level. With a simple illustrative example of an infectious disease insurance, we revisit the two following plans:

1. Annuity for Hospitalization (AH) Plan

Every participant in the mutual insurance fund purchases the coverage by means of an annuity. Insureds in rural areas are charged lower premiums proportional to their reduced susceptibility. From the time of policy issue to the end of the epidemic, insureds can claim a medical examination fee of $\$ 100,000$, once diagnosed with suspicious symptoms, plus hospitalization expenses of $\$ 100,000$ per day, in the form of a life annuity for the period under medical treatment in hospital. Specified beneficiaries are entitled to a death benefit of $\$ 100,000$ if the insured's death is due to the infectious disease. The protection ends at the earliest of the insured's time of death or the end of the epidemic. 


\section{Lump Sum for Hospitalization (SH) Plan}

This plan provides all of the same benefits as in the previous AH plan, with the life annuity being replaced by a lump sum payment of $\$ 100,000$ when the insured is diagnosed positive with the disease. The protection also ends here at the earliest of the policy-holder's death or the end of the epidemic.

The discounted total benefits and premiums in Table 4 are calculated under the assumption that all Hong Kong residents during the pandemic participate in the fund. This yields surprisingly low net level premiums, determined for both plans by the equivalence principle. This reinforces our assertion that a fairly low cost insurance plan could cushion the high impact such pandemics would have on our health care systems when they occur.

\begin{tabular}{|c|c|c|c|}
\hline Plan & P.V. Benefits & P.V. Premiums & Level Premium \\
\hline AH & $3.0571 \times 10^{8}$ & $1.71604 \times 10^{8}$ & 1.78 \\
\hline SH & $1.3231 \times 10^{8}$ & $1.71604 \times 10^{8}$ & 0.77 \\
\hline
\end{tabular}

Table 4: SARS insurance premium rating (per $\$ 100,000$ unit of benefits)

\section{$7 \quad$ Future work}

Research in this emerging type of insurance is at the infancy stage. More work is needed to generalize models that could fit different aspects and features of other pandemics. There have been numerous and extensive studies in epidemiological stochastic modeling. We envision that some of these stochastic models can be incorporated in a more pragmatic way for actuarial applications.

\section{Appendix}

\section{A1 Proof of Proposition 3.1}

From (3.1) and (3.2), we obtain that

$$
s^{\prime}(t)+i^{\prime}(t)=-\alpha i(t), \quad t \geq 0 .
$$

Integrating from 0 to a fixed $t$ gives

$$
s(t)+i(t)-1=-\alpha \int_{0}^{t} i(r) d r, \quad t \geq 0 .
$$


Multiplying both sides by $e^{-\delta t}$ and integrating with respect to $t$, from 0 to $\infty$, yields

$$
\int_{0}^{\infty} e^{-\delta t} s(t) d t+\int_{0}^{\infty} e^{-\delta t} i(t) d t-\frac{1}{\delta}=-\alpha \int_{0}^{\infty} e^{-\delta t} \int_{0}^{t} i(r) d r d t, \quad t \geq 0 .
$$

Exchanging the order of integrals and using the integration by parts gives

$$
\begin{aligned}
\int_{0}^{\infty} \exp (-\delta t) \int_{0}^{t} i(r) d r d t & =-\frac{1}{\delta} \int_{0}^{\infty} \int_{0}^{t} i(r) d r d(\exp (-\delta t)) \\
& =\frac{1}{\delta} \int_{0}^{\infty} \exp (-\delta r) i(r) d r=\frac{1}{\delta} \bar{a}_{\infty}^{i}
\end{aligned}
$$

with the fact that

$$
\lim _{t \rightarrow \infty} e^{-\delta t} \int_{0}^{t} i(r) d r \leq \lim _{t \rightarrow \infty} t e^{-\delta t}=0
$$

Hence (3.9) is obtained upon rearrangement.

\section{A2 Proof of Proposition 3.2}

Substituting (3.1) into (3.11), we have that

$$
\bar{A}_{\bar{\infty}}^{i}=-\int_{0}^{\infty} e^{-\delta t} s^{\prime}(t) d t=s(0)-\delta \int_{0}^{\infty} e^{-\delta t} s(t) d t=s_{0}-\delta \bar{a}_{\bar{\infty}}^{s} .
$$

It follows from (3.9) that

$$
\bar{A}_{\bar{\infty}}^{i}=(\delta+\alpha) \bar{a}_{\infty 1}^{i}-1+s_{0} .
$$

\section{A3 Proof of Proposition 5.1}

Since $s(t)$ and $i(t)$ are all non-negative, from (3.1) and (3.3) we know that $s^{\prime}(t)=-\beta s(t) i(t)<$ 0 , for $t>0$, and $r^{\prime}(t)=\alpha i(t)>0$. Hence $s$ is a monotonically decreasing function and $r$ is monotonically increasing.

If $s_{0} \leq \alpha / \beta$, then $i^{\prime}(t)=i(t)[\beta s(t)-\alpha]<0$, which means that $i(t)$ is monotonically decreasing. If $s_{0}>\alpha / \beta$, because $s$ is monotonically decreasing, then $i^{\prime}(t)=i(t)[\beta s(t)-$ $\alpha]>0$, as long as $s(t)>\alpha / \beta$. Thus $i(t)$ reaches a local maximum at the point $t^{*}$ where $s\left(t^{*}\right)=\alpha / \beta$. As $s(t)$ continues to decrease after reaching $\alpha / \beta$, we must have $i^{\prime}(t)<0$ and hence $i(t)$ is monotonically decreasing thereafter. 


\section{A4 Proof of Proposition 5.2}

To check the concavity of $V$, consider $V^{\prime \prime}(t)$ :

$$
\begin{aligned}
V^{\prime \prime}(t) & =\pi s^{\prime}(t)-i^{\prime}(t)=-\beta \pi s(t) i(t)-\beta s(t) i(t)+\alpha i(t) \\
& =i(t)[\alpha-\beta(\pi+1) s(t)] .
\end{aligned}
$$

It follows that when

$$
\pi>\frac{\alpha}{\beta s(t)}-1, \quad \text { for all } t>0
$$

$V^{\prime \prime}(t)<0$ and hence $V^{\prime \prime}(t)$ is concave. Since $s$ is monotonically decreasing, thus condition (5.1) is required. Similarly, we derive the conditions (5.2) and (5.4) according to the changes in the sign of (7.1). Since the point of inflection $t_{f}$ is where $V(t)$ changes from concave to convex, then it is determined by

$$
V^{\prime \prime}\left(t_{f}\right)=i\left(t_{f}\right)\left[\alpha-\beta(\pi+1) s\left(t_{f}\right)\right]=0,
$$

which implies the condition (5.3).

\section{A5 Proof of Proposition 5.3}

To ensure that $V^{\prime}(t)>0$, we need

$$
\pi>\frac{i(t)}{s(t)}, \quad \text { for all } t
$$

or equivalently,

$$
\ln \pi>\ln i(t)-\ln s(t), \quad \text { for all } t .
$$

Let $f(t)=\ln i(t)-\ln s(t)$, then

$$
f^{\prime}(t)=\frac{i^{\prime}(t)}{i(t)}-\frac{s^{\prime}(t)}{s(t)}=\beta[s(t)+i(t)]-\alpha, \quad \text { by (2.1) and (2.2). }
$$

Since $s(t)+i(t)=1-r(t)$ is monotonically decreasing, we see that $f^{\prime}(t)$ changes from positive to negative at time $t_{m}$, when

$$
s\left(t_{m}\right)+i\left(t_{m}\right)=\alpha / \beta
$$

and $f(t)$ reaches its maximum at time $t_{m}$. Thus, in order for (7.2) to hold $\pi$ must satisfy

$$
\pi>\frac{i\left(t_{m}\right)}{s\left(t_{m}\right)}
$$


Now, since

$$
\frac{i^{\prime}(t)}{s^{\prime}(t)}=\frac{(\beta s(t)-\alpha) i(t)}{-\beta s(t) i(t)}=-1+\frac{\alpha}{\beta s(t)},
$$

integrating both sides to find the orbits of the $(s, i)$-plane, gives:

$$
i(t)+s(t)-\frac{\alpha}{\beta} \ln s(t)=c,
$$

where $c$ is a constant of integration for each specific orbit, say $c=i_{0}+s_{0}-\alpha / \beta \ln \left(s_{0}\right)$. Combining (7.3) and (7.5), we can solve for $s(t)$ and $i(t)$. The solutions are given by

$$
\begin{aligned}
& s\left(t_{m}\right)=\exp \left(1-\frac{\beta c}{\alpha}\right), \\
& i\left(t_{m}\right)=\frac{\alpha}{\beta}-\exp \left(1-\frac{\beta c}{\alpha}\right) .
\end{aligned}
$$

Substituting (7.6) and (7.7) into (7.4) gives condition (5.5).

\section{References}

[1] Anderson, Roy M. and May, Robert M. (1991). Infectious Diseases of Humans. Oxford: Oxford University Press.

[2] Barnes, Belinda and Fulford, Glenn Robert (2008). Mathematical Modelling with Case Studies: A Differential Equations Approach using Maple and MATLAB, 2nd Ed. Boca Raton: Chapman \& Hall/CRC.

[3] Boyce, William E. and DiPrima, Richard C. (1986). Elementary Differential Equations and Boundary Value Problems. 4th Ed. New York: John Wiley and Sons.

[4] Bowers, Newton L., Gerber, Hans U., Hickman, James C., Jones, Donald A. and Nesbitt Cecil J. (1997). Actuarial Mathematics. Chicago: The Society of Actuaries.

[5] Brauer, Fred and Castillo-Chávez, Carlos (2001). Mathematical Models in Population Biology and Epidemiology. Springer-Verlag New York.

[6] Chen, Hua and Cox, Samuel H. (2007). An option-based operational risk management on pandemics. Actuarial Research Clearing House. 2008.1: 1-27.

[7] CIA (2009), Considerations for the development of a pandemic scenario. Canadian Institute of Actuaries. Committee on Risk Management and Capital Requirements. Research Paper 209095. 
[8] Chowell, Gerardo, Fenimore, Paul W., Castillo-Garsow, Mellisa A. and Castillo-Chávez, Carlos (2003). SARS outbreaks in Ontario, Hong Kong and Singapore: the role of diagnosis and isolation as a control mechanism. Journal of Theoretical Biology. 224: 1-8.

[9] Coombes, Kevin R., Hunt, Brian R., Lipsman, Ronald L., Osborn, John E. and Stuck Garrett J. (1997). Differential Equations with Maple. 2nd Ed. New York: John Wiley and Sons.

[10] Cornall, Monica, Chan, Margaret and Sparks Jan (2003). UK vaccination programme risk and reward. The Staple Inn Actuarial Society. London.

[11] Hethcote, Herbert W., Stech, Harlan W. and van den Driessche, Pauline (1981). Periodicity and stability in epidemics models: A survey, in Differential Equations and Applications in Ecology, Epidemics and Population Problems. New York: Academic Press, 65-82.

[12] Hethcote, Herbert W. (2000). The mathematics of infectious diseases. Society for Industrial and Applied Mathematics Review. 42(4): 599-653.

[13] Hoem, Jan M. (1988). The versatility of the Markov chain as a tool in the mathematics of life insurance. Record of Proceedings, International Congress of Actuaries. Helsinki, Finland. 171-202.

[14] JiA, Na, Tsui, Lawrence (2005). Epidemic modelling using SARS as a case study. North American Actuarial Journal. 9(4): 28-42.

[15] Jones, Bruce L. (1994). Actuarial calculations using a Markov model. Transactions of the Society of Actuaries. XLVI: 227-250.

[16] Mäkinen, Mika (2009). Pandemic. International Actuarial Association. Task Force on Mortality. http://www.actuaries.org/CTTEES_TFM/Documents/Pandemic_Makinen_EN.pdf

[17] Mollison, Denis, Isham, Valerie and Grenfell, Bryan T. (1994). Epidemics: models and data. Journal of Royal Statistical Society A. 157(Part 1): 115-149.

[18] Raggett, Graham F. (1982). Modeling the Eyam plague. Bulletin of the Institute of Mathematics and its Applications. 18: 221-226.

[19] Stracke, Andrea and Heinen, Winfried (2006). Influenza pandemic: The impact on an insured lives life insurance portfolio. The Actuary Magazine. June. 
[20] Waters, Howard R. (1984). An approach to the study of multiple state models. Journal of the Institute of Actuaries. 111(Part II): 611-624.
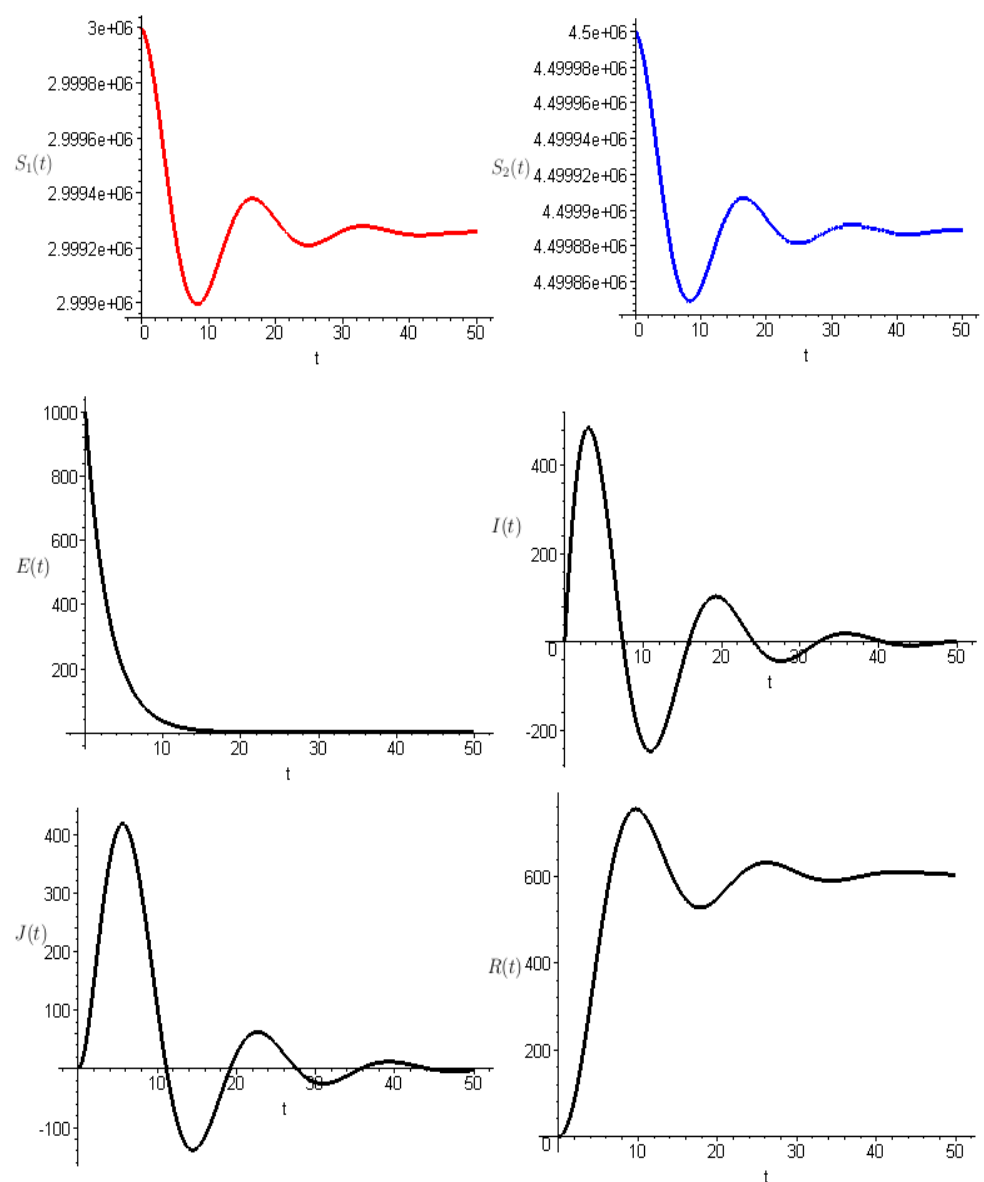

Figure 9: Individual numbers in each compartment: $S_{1}(t), S_{2}(t), E(t), I(t), J(t)$ and $R(t)$. 


\section{List of Recent Technical Reports}

77. Qihe Tang, The Finite Time Ruin Probability of the Compound Poisson Model with Constant Interest Force, June 2005

78. Marc J. Goovaerts, Rob Kaas, Roger J.A. Laeven, Qihe Tang and Raluca Vernic, The Tail Probability of Discounted Sums of Pareto-Like Losses in Insurance, August 2005

79. Yogendra P. Chaubey and Haipeng Xu, Smooth Estimation of Survival Functions under Mean Residual Life Ordering, August 2005

80. Xiaowen Zhou, Stepping-Stone Model with Circular Brownian Migration, August 2005

81. José Garrido and Manuel Morales, On the Expected Discounted Penalty Function for Lévy Risk Processes, November 2005

82. Ze-Chun Hu, Zhi-Ming Ma and Wei Sun, Extensions of Lévy-Khintchine Formula and Beurling-Deny Formula in Semi-Dirichlet Forms Setting, February 2006

83. Ze-Chun Hu, Zhi-Ming Ma and Wei Sun, Formulae of Beurling-Deny and Lejan for Non-Symmetric Dirichlet Forms, February 2006

84. Ze-Chun Hu and Wei Sun, A Note on Exponential Stability of the NonLinear Filter for Denumerable Markov Chains, February 2006

85. H. Brito-Santana, R. Rodríguez-Ramos, R. Guinovart-Díaz, J. BravoCastillero and F.J. Sabina, Variational Bounds for Multiphase Transversely Isotropic Composites, August 2006

86. José Garrido and Jun Zhou, Credibility Theory for Generalized Linear and Mixed Models, December 2006

87. Daniel Dufresne, José Garrido and Manuel Morales, Fourier Inversion Formulas in Option Pricing and Insurance, December 2006

88. Xiaowen Zhou, A Superprocess Involving Both Branching and Coalescing, December 2006 
89. Yogendra P. Chaubey, Arusharka Sen and Pranab K. Sen, A New Smooth Density Estimator for Non-Negative Random Variables, January 2007

90. Md. Sharif Mozumder and José Garrido, On the Relation between the Lévy Measure and the Jump Function of a Lévy Process, October 2007

91. Arusharka Sen and Winfried Stute, A Bi-Variate Kaplan-Meier Estimator via an Integral Equation, October 2007

92. C. Sangüesa, Uniform Error Bounds in Continuous Approximations of Nonnegative Random Variables Using Laplace Transforms, January 2008

93. Yogendra P. Chaubey, Naâmane Laib and Arusharka Sen, A Smooth Estimator of Regression Function for Non-negative Dependent Random Variables, March 2008

94. Alejandro Balbás, Beatriz Balbás and Antonio Heras, Optimal Reinsurance with General Risk Functions, March 2008

95. Alejandro Balbás, Raquel Balbás and José Garrido, Extending Pricing Rules with General Risk Functions, April 2008

96. Yogendra P. Chaubey and Pranab K. Sen, On the Selection of the Smoothing Parameter in Poisson Smoothing of Histogram Estimator: Computational Aspects, December 2008

97. Runhuan Feng and José Garrido, Actuarial Applications of Epidemiological Models, December 2008

Copies of technical reports can be requested from:

Dr. Wei Sun

Department of Mathematics and Statistics

Concordia University

1455 de Maisonneuve Blvd. West,

Montreal, QC, H3G 1M8 CANADA 